\title{
Holographic approach to Drell-Yan process in p-p collisions
}

\author{
Alfonso Ballon-Bayona \\ Centre for Particle Theory, \\ Durham University, \\ Science Laboratories, \\ South Road, \\ Durham DH1 3LE, \\ United Kingdom. \\ E-mail: c.a.m.ballonbayona@durham.ac.uk
}

\author{
Henrique Boschi-Filho*, Nelson R. F. Braga \\ Instituto de Física, \\ Universidade Federal do Rio de Janeiro, \\ Cidade Universitária, \\ Ilha do Fundão, C.P. 68528 , \\ 21941-972 Rio de Janeiro, \\ Brazil. \\ E-mail: boschi@if.ufrj.br, bragadif.ufrj.br
}

\begin{abstract}
We use the holographic hardwall model to study the Drell-Yan process in proton-proton collisions. Describing the hadronic interactions by the exchange of vector mesons, the dilepton angular parameters $\lambda, \mu, v$ are estimated for small transverse momentum $q_{T}^{2}<<Q^{2}$. In this regime, the effective coupling of perturbative QCD is large due to logarithm corrections. We choose kinematical regimes in the region measured at the FNAL E866/NuSea Collaboration and also compare our results with the predictions of perturbation theory.
\end{abstract}

7th International Conference on Mathematical Methods in Physics

April 16-20, 2012

Rio de Janeiro, Brazil

\footnotetext{
* Speaker.
} 
The angular distribution of dileptons produced in proton-proton collisions (Drell-Yan process) was measured recently at the FNAL E866/NuSea Collaboration [1]. We propose a holographic model to estimate the properties of this process (for the details of this presentation see [2]). In our model a virtual photon (which gives rise to the dilepton) comes from the decay of a vector meson, according to vector meson dominance. This vector meson is produced by the annihilation of two other vector mesons emitted by the protons. We use the AdS/QCD hard wall model $[3,4]$ to decribe baryons and vector mesons and their interactions. Hadrons correspond to modes of a Kaluza-Klein expansion of five dimensional fields living in an AdS slice. The size of the slice represents a mass gap in the 4-d effective theory. The hadronic masses are determined by the five dimensional wave functions with appropriate boundary conditions while the effective coupling constants arise from the integration of interaction terms in the 5-d action. These masses and couplings are used to calculate the scattering amplitude relevant for the process of dilepton production. For simplicity, we consider only final hadronic states with spin $1 / 2$. Using this model we find the angular distribution parameters $\lambda, \mu, v$, for kinematical regimes where the dilepton transverse momenta are small. Our results for these parameters are shown in Figure 1 and in Table 1 and compared with predictions from perturbation theory. See the text below for details.

Perturbative QCD calculations for the Drell-Yan cross section work well when the transverse momentum of the dilepton is large with respect to the dilepton mass. However, when the transverse momentum is small, the perturbative series involves the product of the (small) coupling constant $\alpha_{S}$ with large logarithm corrections. This combination leads to a series with a strong effective coupling. In this case one needs a resummation to all orders since the standard perturbative approach is not reliable in this regime [5].

Recently, alternative approaches to gauge theories at strong coupling were developed based on gauge string dualities inspired in the AdS/CFT correspondence. In particular these dualities lead to some holographic models to describe non perturbative aspects of QCD known as AdS/QCD. For reviews see for instance [6-9].

We fix the size of the hard wall AdS slice $z_{0}=1 / \Lambda$, by fitting the mass of the $\rho$ meson $m_{\rho}=0.776 \mathrm{GeV}$. This gives $\Lambda=0.323 \mathrm{GeV}$. In the frame of the center of mass of the final hadrons, one can write the protons and the photon momenta as

$$
\begin{aligned}
& P_{1}=\left(\sqrt{p^{2}+M_{1}^{2}}, 0,0, p\right) ; \quad q=\left(\sqrt{m_{\gamma}^{2}+q_{2}^{2}+q_{3}^{2}}, 0, q_{2}, q_{3}\right) \\
& P_{2}=\left(\sqrt{q_{2}^{2}+\left(q_{3}-p\right)^{2}+M_{1}^{2}}, 0, q_{2}, q_{3}-p\right),
\end{aligned}
$$

where $M_{1}=3.83 \Lambda=1.2 \mathrm{GeV}$ is the "proton" mass in the model, once the infrared scale was fixed by the mass of the $\rho$ meson. We analysed different kinematical regimes, contained in the region of the experimental data of ref. [1]. Each kinematical regime is defined by a choice of the values of $p, q_{2}, q_{3}$ and $m_{\gamma}$. From these quantities we obtain the corresponding values for $\sqrt{s}, q_{T}$ and $x_{F} \equiv 2 \frac{q_{p}}{\sqrt{s}}$ where

$$
q_{p}=\frac{q \cdot\left(P_{1}-P_{2}\right)}{\sqrt{s}} \quad, \quad q_{P}=-\frac{q \cdot\left(P_{1}+P_{2}\right)}{\sqrt{s}} \quad, \quad q_{T}=\left(q_{P}^{2}-q_{p}^{2}-m_{\gamma}^{2}\right)^{1 / 2} .
$$

The numerical calculation was performed using the "Mathematica" package. We computed the diagonal elements of the hadronic tensor $W^{\mu \nu}$ (see eq. (9) below). Using these results it is 

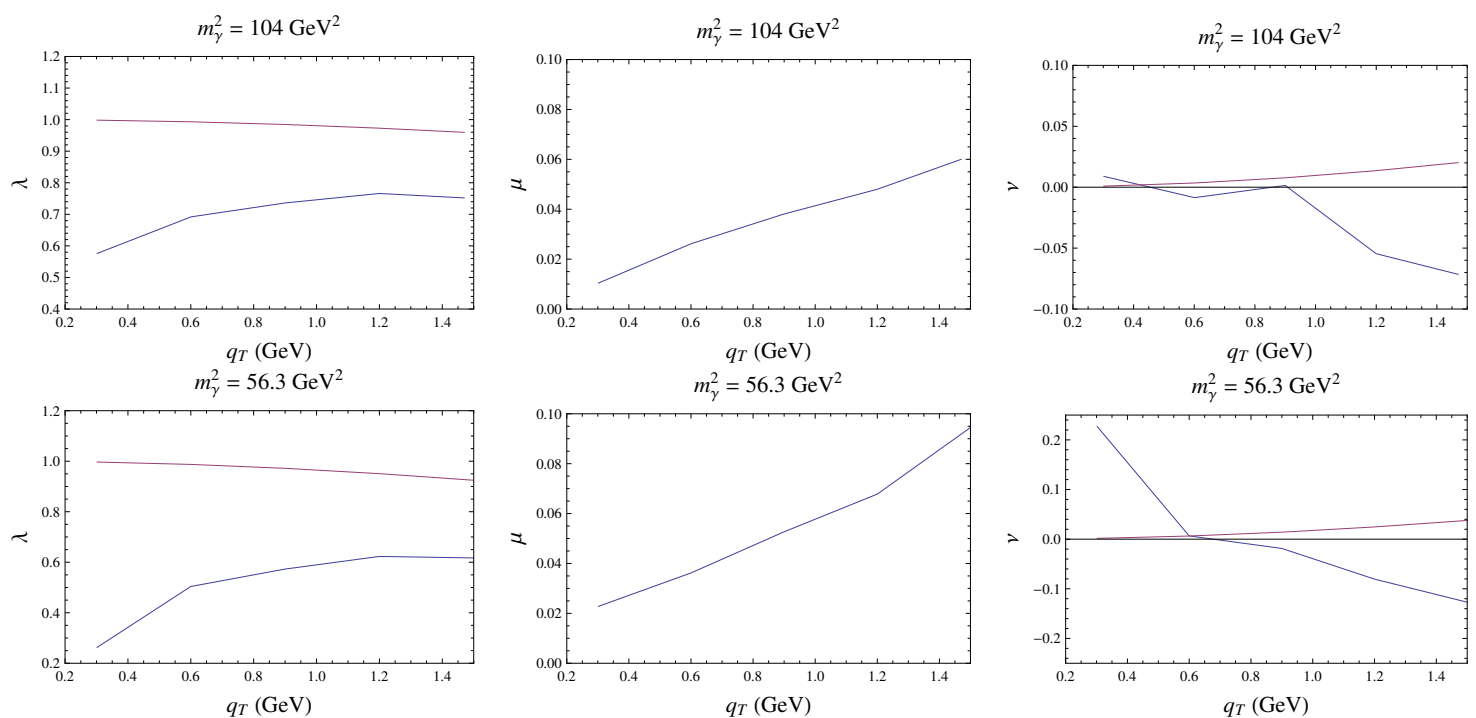

Figure 1: We plot our results (blue lines) and the perturbative predictions (red lines) for the angular parameters $\lambda, \mu, v$ for $m_{\gamma}^{2}=104 \mathrm{GeV}^{2}$ and $m_{\gamma}^{2}=56.3 \mathrm{GeV}^{2}$. Note that $\mu$ is not compared with the corresponding perturbative result $\mu_{\text {pert }}$ since the later it is not known, thanks to its dependence on the unknown parton distribution functions [5].

possible obtain the helicity structure functions from which we calculate the angular parameters $\lambda, \mu, v$ defined in eq. (14) in the Appendix. In Table 1 we show our choices of kinematical regimes and the corresponding results for the angular parameters from our model and from the perturbative expressions. For a more detailed discussion of the results presented here see [2].

The hard wall model consists on a 5-d theory living in an $\mathrm{AdS}_{5}$ slice with metric [3, 4]

$$
d s^{2}=\frac{R^{2}}{z^{2}}\left[-d t^{2}+d \vec{x}^{2}+d z^{2}\right]
$$

where $0<z<z_{0}=1 / \Lambda$ and $\Lambda$ is an IR energy scale for the dual 4-d effective theory. The physical spectrum of the hadronic particles is obtained after imposing boundary conditions at $z=z_{0}$.

Considering the action for the non-Abelian 5-d gauge fields in the presence of a gauge-fixing term and expanding it as a Kaluza-Klein tower we obtain a 4-d effective action [2]

$$
S=\int d^{4} x \sum_{n=1}^{\infty} \operatorname{Tr}\left\{\frac{1}{2} \tilde{v}_{\mu}^{n}\left[\eta^{\mu v}\left(\partial^{2}-m_{n}^{2}\right)+\left(\frac{1}{\xi}-1\right) \eta^{\mu \sigma} \eta^{v \rho} \partial_{\sigma} \partial_{\rho}\right] \tilde{v}_{v}^{n}+g_{v^{n}} \eta^{\mu v} \tilde{v}_{\mu}^{n} a_{v}\right\}+\ldots
$$

where the dots represent interactions and divergent terms. The fields $\tilde{v}_{\mu}^{n}$ are interpreted as vector mesons with mass $m_{n}$ while the field $a_{\mu}$ is interpreted as the photon which couples to vector mesons with the coupling constant $g_{v^{n}}$. This way the hard wall model realizes vector meson dominance.

The interaction Lagrangian for three vector mesons is given by [2]

$$
\begin{aligned}
S_{V V V} & =-\kappa \int d^{4} x d z \sqrt{-g} h^{2} \eta^{\mu \alpha} \eta^{v \beta} \operatorname{Tr}\left\{\left[A_{\mu}, A_{v}\right] \partial_{\alpha} A_{\beta}\right\} \\
& =-\sum_{n, m, \ell} g_{v^{n} v^{m_{v}}} \int d^{4} x \eta^{\mu \alpha} \eta^{v \beta} \operatorname{Tr}\left\{\left[\tilde{v}_{\mu}^{n}, \tilde{v}_{v}^{m}\right] \partial_{\alpha} \tilde{v}_{\beta}^{\ell}\right\}+\ldots
\end{aligned}
$$


Table 1: Kinematical variables $\left(p, q_{2}, q_{3}, \sqrt{s}, q_{T}\right.$ in GeV) and angular parameters calculated from our model and from the perturbative approach, for $m_{\gamma}^{2}=104 \mathrm{GeV}^{2}$ (upper part) and for $m_{\gamma}^{2}=56.3 \mathrm{GeV}^{2}$ (lower part).

\begin{tabular}{cccccc|ccc|cc}
\hline \multicolumn{7}{c|}{ Kinematical regimes } & \multicolumn{3}{c|}{ Our results } & \multicolumn{2}{c}{ Perturb. results } \\
\hline$p$ & $q_{2}$ & $q_{3}$ & $\sqrt{s}$ & $q_{T}$ & $x_{F}$ & $\lambda$ & $\mu$ & $v$ & $\lambda_{\text {pert }}$ & $v_{\text {pert }}$ \\
\hline 25.8 & 1.61 & 11.3 & 38.8 & 1.47 & 0.373 & 0.75 & 0.060 & -0.072 & 0.960 & 0.0201 \\
25.6 & 1.29 & 10.8 & 38.9 & 1.20 & 0.361 & 0.77 & 0.048 & -0.055 & 0.973 & 0.0136 \\
24.8 & 0.937 & 9.69 & 38.9 & 0.900 & 0.331 & 0.74 & 0.038 & 0.0014 & 0.985 & 0.00770 \\
24.1 & 0.565 & 8.40 & 38.9 & 0.601 & 0.294 & 0.69 & 0.026 & -0.0086 & 0.993 & 0.00346 \\
22.7 & 0.162 & 6.02 & 38.9 & 0.302 & 0.218 & 0.58 & 0.010 & 0.0089 & 0.998 & 0.000876 \\
\hline 25.8 & 1.53 & 11.3 & 38.9 & 1.50 & 0.398 & 0.62 & 0.095 & -0.13 & 0.925 & 0.0377 \\
25.5 & 1.20 & 10.8 & 38.9 & 1.20 & 0.386 & 0.62 & 0.068 & -0.081 & 0.951 & 0.0246 \\
24.8 & 0.859 & 9.69 & 38.9 & 0.900 & 0.355 & 0.57 & 0.053 & -0.019 & 0.972 & 0.0141 \\
24.1 & 0.501 & 8.40 & 38.9 & 0.600 & 0.316 & 0.50 & 0.036 & 0.0066 & 0.987 & 0.00633 \\
22.6 & 0.0808 & 6.02 & 38.9 & 0.302 & 0.236 & 0.26 & 0.023 & 0.23 & 0.997 & 0.00162 \\
\hline
\end{tabular}

In order to describe spin 1/2 states within the hard wall model, we start with a 5-d Dirac action. Considering a Kaluza-Klein expansion for the fermionic fields we obtain the 4-d effective action

$$
S_{F}=\sum_{n=1}^{\infty} \int d^{4} x \bar{u}^{n}(x)\left[i \eta^{\mu v} \gamma_{\mu} \partial_{v}-M_{n}\right] u^{n}(x)
$$

where $M_{n}$ are the masses of the baryonic states of spin $1 / 2$ in the 4-d theory represented by the spinors $u^{n}(x)$ and $\vec{u}^{n}(x) . M_{1}$ is identified with the proton mass and $M_{n}$ with $n=2,3, \ldots$, correspond to excited states. We can describe the interaction of two fermions and one vector meson considering the 5-d action

$$
S_{F F V}=\kappa_{F} \int d^{4} x d z \sqrt{-g} \frac{z}{R} \eta^{\mu v} \bar{\psi} \gamma_{\mu} A_{\nu} \psi
$$

that comes from imposing invariance of the 5-d fermionic action with respect to 4-d gauge transformations. Using the Kaluza-Klein expansions for the fields we find (using the notation of [2])

$$
S_{F F V}=\sum_{n, m, \ell} \int d^{4} x \eta^{\mu v} \bar{u}^{n} \gamma_{\mu}\left[g_{\bar{u}^{n} v^{m} u^{\ell}}^{+} \mathscr{P}_{+}+g_{\bar{u}^{n} v^{m} u^{\ell}}^{-} \mathscr{P}_{-}\right] \tilde{v}_{v}^{m} u^{\ell}+\ldots
$$

We calculate the contribution to dilepton production at three level. This corresponds to the interaction of two protons with momenta $P_{1}$ and $P_{2}$ through the exchange of vector mesons $v^{n}$ and $v^{m}$. These vector mesons combine into another vector meson $v^{\ell}$ that decays into a time-like photon that eventually gives rise to a lepton pair. At lowest order, the final state corresponding to each proton is one excited baryon. These final baryons are not measured, so the hadronic tensor $W^{\mu \nu}$ involves the sum over all possible baryonic states $X$ and $Y$. For simplicity, we consider that each final state $X$ and $Y$ contain just one spin 1/2 baryon. 
The optical theorem relates the hadronic tensor to the imaginary part of the amplitude. Using the Feynman rules coming from the 4-d effective Lagrangians of the hardwall model we get the hadronic tensor [2]

$$
\begin{aligned}
W^{\mu v} & =\frac{1}{4\left(2 \pi^{2}\right)} f^{a b c} f^{a b c} \sum_{m, n} \sum_{\bar{m}, \bar{n} n_{X}, n_{Y}} \int \frac{d^{3} \vec{P}_{X}}{2 \sqrt{\vec{P}_{X}^{2}+M_{n_{X}}^{2}}} \int \frac{d^{3} \vec{P}_{Y}}{2 \sqrt{\vec{P}_{Y}^{2}+M_{n_{Y}}^{2}}} \\
& \times \delta^{4}\left(P_{1}+P_{2}-P_{X}-P_{Y}-q\right) \sum_{S_{H 1}}\left(\bar{u}_{(1)}^{1} \Gamma_{\bar{\alpha}}\left(\bar{n}, n_{X}\right)\left[\gamma_{\lambda} P_{X}^{\lambda}+M_{n_{X}}\right] \Gamma_{\alpha}\left(n, n_{X}\right) u_{(1)}^{1}\right) \\
& \times \sum_{S_{H 2}}\left(\bar{u}_{(2)}^{1} \Gamma_{\bar{\beta}}\left(\bar{m}, n_{Y}\right)\left[\gamma_{\sigma} P_{Y}^{\sigma}+M_{n_{Y}}\right] \Gamma_{\beta}\left(m, n_{Y}\right) u_{(2)}^{1}\right) \\
& \times \Delta^{\alpha \alpha^{\prime}}\left(P_{1}-P_{X}, m_{n}^{2}\right) \Delta^{\bar{\alpha} \bar{\alpha}^{\prime}}\left(P_{1}-P_{X}, m_{\bar{n}}^{2}\right) \Delta^{\beta \beta^{\prime}}\left(P_{2}-P_{Y}, m_{m}^{2}\right) \Delta^{\bar{\beta} \bar{\beta}^{\prime}}\left(P_{2}-P_{Y}, m_{\bar{m}}^{2}\right) \\
& \times \mathscr{C}_{\alpha^{\prime} \beta^{\prime} \tau} \mathscr{C}_{\bar{\alpha}^{\prime} \bar{\beta}^{\prime} \bar{\tau}} \sum_{\ell} g_{v^{n} v^{m} v^{\prime} \ell} g_{v^{\ell}} \Delta^{\mu \tau}\left(q, m_{\ell}\right) \sum_{\bar{\ell}} g_{v^{\bar{n}} v^{\prime} \bar{m}_{v} \bar{\ell}} g_{v^{\ell}} \Delta^{\mu \bar{\tau}}\left(q, m_{\bar{\ell}}^{2}\right)
\end{aligned}
$$

where $u_{(1)}^{1}$ and $u_{(2)}^{1}$ are the spinors representing the initial protons with momenta $P_{1}$ and $P_{2}$,

$$
\begin{aligned}
\Gamma_{\alpha}\left(n, n_{X}\right) & =\gamma_{\alpha}\left[g_{\bar{u}^{n} X v^{n} u^{1}}^{+} \mathscr{P}_{+}+g_{\bar{u}^{n} X v^{n} u^{1}}^{-} \mathscr{P}_{-}\right] \\
\Delta^{\sigma \rho}\left(P, m_{i}^{2}\right) & =\frac{1}{P^{2}+m_{i}^{2}}\left[\eta^{\sigma \rho}-(1-\xi) \frac{P^{\sigma} P^{\rho}}{P^{2}+\xi m_{i}^{2}}\right] \\
\mathscr{C}_{\alpha^{\prime} \beta^{\prime} \tau} & =\eta_{\alpha^{\prime} \beta^{\prime}}\left(P_{1}-P_{X}-P_{2}+P_{Y}\right)_{\tau}+\eta_{\beta^{\prime} \tau}\left(P_{2}-P_{Y}+q\right)_{\alpha^{\prime}}-\eta_{\alpha^{\prime} \tau}\left(P_{1}-P_{X}+q\right)_{\beta^{\prime}}
\end{aligned}
$$

and $\eta_{\alpha \beta}=\operatorname{diag}(-,+,+,+)$ is the Minkowski flat spacetime metric. Note that the denominators of the fermionic propagators do not appear in eq. (9) since they are transformed into delta functions when one takes the imaginary part of the forward scattering tensor. These delta functions impose on-shell conditions on the final baryonic momenta $P_{X}=\left(E_{X}, \vec{P}_{X}\right)$ and $P_{Y}=\left(E_{Y}, \vec{P}_{Y}\right)$, implying that $E_{X}=\sqrt{\vec{P}_{X}^{2}+M_{n_{X}}^{2}}$ and $E_{Y}=\sqrt{\vec{P}_{Y}^{2}+M_{n_{Y}}^{2}}$.

\section{Appendix: Dilepton production in P-P collisions}

The differential cross section for dilepton production in $\mathrm{p}$-p collisions can be written as (for the unpolarized case)

$$
d \sigma=\frac{e^{4}}{\left(q^{2}\right)^{2} s} W^{\mu v} L_{\mu v} \frac{d^{3} \vec{k}_{1}}{(2 \pi)^{3} 2\left|\vec{k}_{1}\right|} \frac{d^{3} \vec{k}_{2}}{(2 \pi)^{3} 2\left|\vec{k}_{2}\right|},
$$

where $s=-\left(P_{1}+P_{2}\right)^{2}$ and $q^{2}=-m_{\gamma}^{2}$ with $m_{\gamma}>0$ the virtual photon mass. $L_{\mu v}$ is the leptonic tensor. The lepton masses were neglected with respect to their momenta $\vec{k}_{1}, \vec{k}_{2}$ and the proton masses were neglected with respect to the center of mass energy $\sqrt{s}$.

The hadronic tensor $W^{\mu v}$ can be decomposed in terms of the helicity structure functions as [5]

$$
\begin{aligned}
W^{\mu v} & =\left(\eta^{\mu v}-\frac{q^{\mu} q^{v}}{q^{2}}\right)\left(W_{T}+W_{\Delta \Delta}\right)-2 X^{\mu} X^{v} W_{\Delta \Delta} \\
& +Z^{\mu} Z^{v}\left(W_{L}-W_{T}-W_{\Delta \Delta}\right)-\left(X^{\mu} Z^{v}+X^{v} Z^{\mu}\right) W_{\Delta},
\end{aligned}
$$

so that the differential cross takes the form (in the photon rest frame)

$$
d \sigma=\frac{e^{4}}{8(2 \pi)^{6} m_{\gamma}^{2} s}\left[W_{T}\left(1+\cos ^{2} \theta\right)+W_{L}\left(1-\cos ^{2} \theta\right)+W_{\Delta} \sin 2 \theta \cos \phi\right.
$$




$$
\left.+W_{\Delta \Delta} \sin ^{2} \theta \cos 2 \phi\right] \sin \theta d \theta d \phi d^{4} q
$$

where $\theta$ and $\phi$ are the spherical angular coordinates for the vector $\vec{k}_{1}$ with respect to the Cartesian system $X, Y, Z: \vec{k}_{1}=\left|\vec{k}_{1}\right|(\sin \theta \cos \phi, \sin \theta \sin \phi, \cos \theta)$.

In order to compare theoretical predictions with experimental results it is interesting to introduce parameters that characterize the angular dependence of the cross section. These parameters are defined by

$$
\lambda=\frac{W_{T}-W_{L}}{W_{T}+W_{L}} \quad, \quad \mu=\frac{W_{\Delta}}{W_{T}+W_{L}} \quad, \quad v=\frac{2 W_{\Delta \Delta}}{W_{T}+W_{L}},
$$

so that

$$
d \sigma \sim 1+\lambda \cos ^{2} \theta+\mu \sin 2 \theta \cos \phi+\frac{v}{2} \sin ^{2} \theta \cos 2 \phi .
$$

Acknowledgements. We would like to thank the organizers of 7th International Conference on Mathematical Methods in Physics, Rio de Janeiro, 2012 for the very nice and collaborative atmosphere created. A.B.-B. is supported by STFC Rolling Grant ST/G000433/1. H.B.-F. and N.R.F.B. are partially supported by Capes and CNPq, Brazilian agencies.

\section{References}

[1] L. Y. Zhu et al. [FNAL E866/NuSea Collaboration], "Measurement of Angular Distributions of Drell-Yan Dimuons in p + p Interactions at 800-GeV/c," Phys. Rev. Lett. 102, 182001 (2009) [arXiv:0811.4589 [nucl-ex]].

[2] C. A. B. Bayona, H. Boschi-Filho and N. R. F. Braga, "Holographic model for dilepton production in p-p collisions," Nucl. Phys. B 851, 66 (2011) [arXiv:1007.4362 [hep-th]].

[3] H. Boschi-Filho and N. R. F. Braga, "Gauge / string duality and scalar glueball mass ratios," JHEP 0305, 009 (2003) [hep-th/0212207].

[4] H. Boschi-Filho and N. R. F. Braga, "QCD / string holographic mapping and glueball mass spectrum," Eur. Phys. J. C 32, 529 (2004) [hep-th/0209080].

[5] D. Boer and W. Vogelsang, "Drell-Yan lepton angular distribution at small transverse momentum," Phys. Rev. D 74, 014004 (2006) [hep-ph/0604177].

[6] H. Boschi-Filho and N. R. F. Braga, "AdS/CFT correspondence and strong interactions," PoS IC 2006, 035 (2006) [hep-th/0610135].

[7] K. Peeters and M. Zamaklar, "The String/gauge theory correspondence in QCD," Eur. Phys. J. ST 152, 113 (2007) [arXiv:0708.1502 [hep-ph]].

[8] J. Erdmenger, N. Evans, I. Kirsch and E. Threlfall, "Mesons in Gauge/Gravity Duals - A Review," Eur. Phys. J. A 35, 81 (2008) [arXiv:0711.4467 [hep-th]].

[9] S. J. Brodsky and G. F. de Teramond, "Applications of AdS/QCD and Light-Front Holography to Baryon Physics," AIP Conf. Proc. 1388, 22 (2011) [arXiv:1103.1186 [hep-ph]]. 